\title{
Simulation of multiphase flows with variable surface tension using the Lattice Boltzmann method
}

\author{
S. Stensholt \\ University of Bergen, Department of Mathematics, Norway
}

\begin{abstract}
Methods for implementing variable surface tension on two popular LatticeBoltzmann models, the original gradient-based chromodynamic model, and the Shan-Chen model, are explored and examined. The experiment, inspired by the work of Greenspan, consists of reducing the surface tension at two poles of a circular droplet due to a diffusive solute (surfactant). Both Lattice Boltzmann models are able to simulate the expected initial deformation where the droplet is stretched along the pole axis, and contracts at the equator. We observed no furrowing to the droplet, which verifies the work of He and Dembo who concluded that variation in surface tension cannot alone account for such furrowing. We were able to simulate the process as the surfactant diffuses over the entire interface and the spatial variation in surface tension vanishes. The droplet reverts back to its original circular shape with the overall surface tension reduced. Variable surface tension is easier to implement with the chromodynamic model. The physically direct Shan-Chen model, which has superior isotropic qualities, can also be used for variable surface tension. However, coordinating the decline of the surface tension with the reduction in the separation forces is a more delicate matter, and the diffusivity of the interface increases if the surface tension is weakened. Keywords: Lattice Boltzmann, variable surface tension, droplet deformation.
\end{abstract}

\section{Introduction}

The study of fluid flow with variable surface tension has seen multiple applications, including biological fields related to cell division [4-6], and oil reservoir dynamics. 
In this article, we apply the Lattice Boltzmann method to study this effect. We consider two popular models, the chromodynamic method, and the Shan-Chen method.

In order to simulate the effects of microemulsions, lattice Boltzmann models with amphiphile surfactants are largely based on the free energy model of Orlandini et al. [7], see for example references [8-11]. The model used here is simpler, since the purpose is primarily to investigate the deformative effects of surface tension rather than the effects on the interface structure. Lattice Boltzmann methods with variable surface tension have also been employed by Farhat and Lee [12] in order to simulate the biconcave shape of red blood cells.

If the surface tension is constant, all droplets will attain a circular shape, but if surface tension varies along the interface, the interface curvature will be greater where the surface tension is low. By manipulating the surface tension, it is possible to create deformations to an initially circular droplet.

In this article we employ an experiment based on that conducted by Greenspan [3], where surface tension-reducing surfactant is applied to two poles of a droplet. The expected deformation is an elongation along the axis and contraction at the equator. Greenspan even demonstrated furrowing to the droplet, although Sapir and Nir [13], He and Dembo [4], and Li and Lubkin [14] were unable to produce deformations as large as this.

Our main goal with conducting this experiment is to demonstrate the validity of our variable surface tension methods. In addition we wish to illustrate the relative ease with which a solute surfactant can be applied in the method.

\section{Lattice Boltzmann method}

The Lattice Boltzmann method was introduced by McNamara and Zanetti [15] as an extension to the lattice gas cellular automata. The method can alternately be derived by discretizing the Boltzmann equation [16]. The first method to extend the method to handle multiphase flows was introduced by Gunstensen et al. [1] in 1991, and an alternate method was proposed by Shan and Chen in 1993 [2].

Our interest is in two component multiphase flow. The components are labelled "red" (superscript $R$ ) and "blue" (superscript $B$ ). An arbitrary component is labelled superscript $s$, while we let $\hat{s}$ represent the other component.

Lattice Boltzmann methods aim to simulate the evolution of the discrete distribution functions $f_{i}^{s}(\boldsymbol{x}, t)$, which tells how many particles of mass 1 are moving along vector $\boldsymbol{e}_{i}$ at site $\boldsymbol{x}$ at time $t$. Summing the distribution function over all $i$ will yield the mass density, summing $f_{i}^{s} \boldsymbol{e}_{i}$ over all $\mathrm{i}$ will yield the particle momentum. As long as we are away from the interface, we use the ideal gas equation

$$
p=\left(\rho^{R}+\rho^{B}\right) c^{2}
$$

for pressure where $c^{2}=\frac{1}{3}$ is the square of the speed of sound. 
We have employed the D2Q9 lattice [17], which has two dimensions and nine possible velocities at each site, given by

$$
\boldsymbol{e}_{\boldsymbol{i}}= \begin{cases}(0,0), & i=0, \\ \left.\left(\cos \frac{(i-1) \pi}{2}, \sin \frac{(i-1) \pi}{2}\right)\right) & i=1, \ldots 4 \\ \sqrt{2}\left(\cos \left[\frac{(i-5) \pi}{2}+\frac{\pi}{4}\right], \sin \left[\frac{(i-5) \pi}{2}+\frac{\pi}{4}\right]\right) & i=5, \ldots 8\end{cases}
$$

The equation to update the distribution function is given by

$$
f_{i}^{s}\left(\boldsymbol{x}+\boldsymbol{e}_{i}, t+1\right)=f_{i}^{s}(\boldsymbol{x}, t)+\Omega\left(f_{i}^{s}, f_{i}^{s_{1}}, f_{i}^{s_{2}}, \ldots\right),
$$

where the first terms handle propagation (streaming) of particles, while the collision term $\Omega$ deals with interparticle collisions, including the generation of surface tension and phase separation.

In the chromodynamic model by Gunstensen, the process is to first create surface tension by increasing the proportion of particles moving normal to the interface, and then enforce phase separation through a recolouring step. Central to this idea is the concept of the color gradient $\boldsymbol{F}$

$$
\boldsymbol{F}(\boldsymbol{x}, t)=\sum_{i=1}^{8} \boldsymbol{e}_{i}\left(\rho^{R}\left(\boldsymbol{x}+\boldsymbol{e}_{i}\right)-\rho^{B}\left(\boldsymbol{x}+\boldsymbol{e}_{i}\right)\right) .
$$

The collision operator $\Omega^{s}$ can be written as $\Omega^{s}=\Omega 3^{s}\left[\Omega 1^{s}+\Omega 2^{s}\right]$ [18], where $\Omega 1^{s}$ is the single relaxation time (BGK [19]) operator

$$
\Omega 1^{s}=-\frac{1}{\tau^{s}}\left(f_{i}^{s}-f_{i}^{s(e q)}\right),
$$

with local equilibrium distribution $f_{i}^{s(e q)}$ given by

$$
f_{i}^{s(e q)}\left(\boldsymbol{u}^{s}\right)=w_{i} \rho\left(1+\frac{3\left(\boldsymbol{e}_{i} \cdot \boldsymbol{u}^{s}\right)}{c^{2}}+\frac{9\left(\boldsymbol{e}_{i} \cdot \boldsymbol{u}^{s}\right)^{2}}{2 c^{4}}-\frac{3}{2} \frac{\left(u^{s}\right)^{2}}{c^{2}}\right)
$$

where $\boldsymbol{u}^{s}$ is the macroscopic velocity of component $s$. The weights $w_{i}$ are $w_{0}=4 / 9, w_{1, \ldots, 4}=1 / 9, w_{5, \ldots 8}=1 / 36$. Surface tension is generated by $\Omega 2$ and is given by [20]

$$
\Omega 2_{i}^{s}=\frac{A}{2}|\boldsymbol{F}|\left(w_{i} \frac{\left(\boldsymbol{e}_{i} \cdot \boldsymbol{F}\right)^{2}}{|\boldsymbol{F}|^{2}}-B_{i}\right), \quad i=0, \ldots, 8,
$$

with $A$ being a parameter for surface tension, and $B_{i}$ are the constants $B_{0}=$ $-4 / 27, B_{1, \ldots, 4}=2 / 27$ and $B_{5, \ldots, 8}=5 / 108$. Surface tension is proportional to the parameter $A$ in (7), and a variation in surface tension can therefore be conveniently applied by adjusting $A$. 
The recolouring algorithm, which accounts for phase segregation, can be applied through the formula given by Latva-Kokko and Rothman [21]

$$
\Omega 3_{i}=\left\{\begin{array}{l}
f_{i}^{R}=\phi^{R} f_{i}+\phi^{R} \phi^{B} f_{i}^{e q}(\rho, 0) \cos \theta, \\
f_{i}^{B}=\phi^{B} f_{i}-\phi^{R} \phi^{B} f_{i}^{e q}(\rho, 0) \cos \theta
\end{array},\right.
$$

where $\phi^{R}$ and $\phi^{B}$ are the fraction of particles which are red and blue respectively.

In the Shan-Chen model, the collision operator is basically the BGK operator (5), but the velocity $\boldsymbol{u}^{s}$ used to calculate $f_{i}^{e q}$ is perturbed to $\boldsymbol{u}^{\prime s}$ by a repulsive force between particles of opposite color

$$
\rho \boldsymbol{u}^{\prime s}=\rho \boldsymbol{u}^{s}-\psi^{s}(\boldsymbol{x}, t) G \sum_{i=1}^{8} w_{i} \psi^{\hat{s}}\left(\boldsymbol{x}+\boldsymbol{e}_{i}, t\right) \boldsymbol{e}_{i},
$$

where $\psi^{s}$ simply can be defined as $\rho^{s}$ in the multicomponent model. The parameter $G$ controls surface tension and phase separation. If $G$ is large enough, the separation forces will overcome the diffusion and yield separate phases. The critical value of $G$ for separation is a function of the proportion of each phase [22].

The Shan-Chen model does not conserve momentum on each individual site as the chromodynamic model does, but if we maintain symmetry between two interacting sites we maintain global conservation of momentum [23]. A practical drawback to the Shan-Chen model is the difficulty in determining the surface tension a priori. The surface tension generally rises with $G$, but it is not a convenient proportional relationship as there was for the chromodynamic model [24].

For both the chromodynamic and Shan-Chen models, solute flow is implemented by introducing a third component with its own distribution function $g$. This component evolves according to an equation like (3), with a relaxation time $\tau^{g}$ which controls its diffusivity, but it does not contribute to the calculation of $\boldsymbol{u}$ [25]. In order to keep the solute on the interface, we implemented a force perturbing the solute's velocity $\boldsymbol{u}$ to $\boldsymbol{u}^{\prime}$ by

$$
\boldsymbol{u}^{\prime}=\boldsymbol{u}+a\left(\sum_{i=1}^{8} w_{i} \phi^{R}\left(\boldsymbol{x}+\boldsymbol{e}_{i}\right) \phi^{B}\left(\boldsymbol{x}+\boldsymbol{e}_{i}\right) \boldsymbol{e}_{i}\right),
$$

where $a$ is a positive constant which we set to 10 . This is large enough to keep almost all the solute on the surface, but not so large that it destabilizes the model.

For both models, the surface tension can be found by measuring the radius $r$ and pressure difference $\Delta p$ between a circular droplet's interior and exterior. The surface tension parameter $\sigma$ is then given by Laplace's law $\sigma=r \Delta p$.

\section{Simulation and discussion}

In our simulation, we started with an initially stable droplet of radius 10.4 in units of grid spacing. For the chromodynamic test, we set the parameter $A=0.10$ while 
we set the parameter $G=2.0$ for the Shan-Chen model. These choices yield almost the same initial pressure difference of $\Delta p=0.122$ for the chromodynamic model and 0.121 for the Shan-Chen model.

At $t=0$ a surfactant solute is applied at two nodes, at opposite ends of the droplet, with an initial concentration of $C=1$. Following the lead of Greenspan, we attempt to let the reduction in surface tension be proportional to the concentration of surfactant, although we do not let it go below zero. The new and reduced parameter for the chromodynamic test is therefore

$$
A(\boldsymbol{x})=\max \left(0, A_{0}-\zeta C(\boldsymbol{x})\right)
$$

where the parameter $\zeta$ controls how effective the solute is at reducing the surface tension.

Reducing the surface tension in the Shan-Chen model is a more delicate matter. As mentioned in section 2, maintaining symmetry between sites is essential, so introducing a spatial variation to the parameter $G$ in equation (9) by

$$
G(\boldsymbol{x})=\max \left(0, G_{0}-\gamma C(\boldsymbol{x})\right) .
$$

would violate the conservation of momentum unless $C(\boldsymbol{x})$ is constant in space. However, we can achieve a result similar to (12) by multiplying the $\psi^{s}$ values in (9) with a factor $d(\boldsymbol{x})$, where $0<d<1$ reduces the surface tension and $d>1$ increases the surface tension. If we set

$$
d(\boldsymbol{x})=\max (0, \sqrt{1-\gamma C(\boldsymbol{x}) / 2})
$$

we have the same result with constant $C$ as (12) would have given us.

As mentioned, $\sigma$ is not proportional to $G$, since much of the separation force is used to overcome diffusion. Chin et al. did however indicate a linear relationship between $G$ and $\sigma$ for moderate $G$, and some experiments we have conducted indicate that $\sigma$ is roughly proportional to $G-1 / \rho$. We therefore estimate that surface tension vanishes when

$$
C \geq \frac{1}{\gamma}\left(G-\frac{1}{\bar{\rho}}\right),
$$

where $\bar{\rho}$ is the average density, equal to 1 in our experiments. We set the parameters $\zeta=2.5$ and $\gamma=25$ so that both these models give zero surface tension for $C \geq 0.04$. The relaxation time $\tau^{g}$ for the solute species is 1 . The evolution of the droplets' shapes and surfactant concentrations are shown in figures 1 and 2 for the tests with the chromodynamic and Shan-Chen models respectively.

In the chromodynamic model, the deformations are relatively small, but are clearly noticeable at $t=150$. When the surfactant is evenly distributed, there is no longer any variation in surface tension, and the droplet reverts back to the original circular shape, as seen for $t=500$. At $t=2000$ (not pictured) the pressure difference has dropped to 0.0107 (we recall that $\Delta p=0.0122$ at $t=0$ ). 

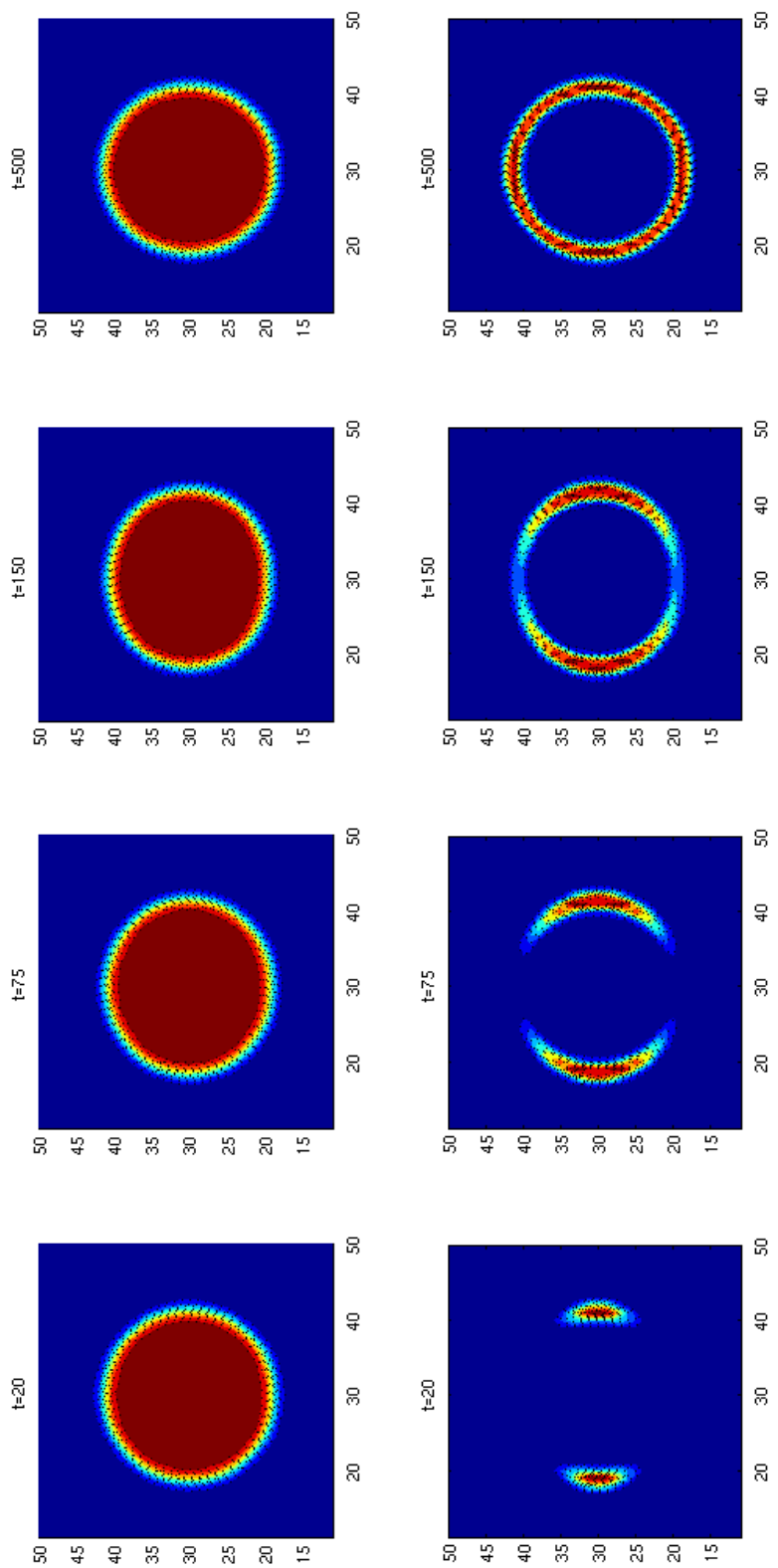

?

更

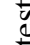

$\stackrel{Ð}{=}$

cे

통

¿

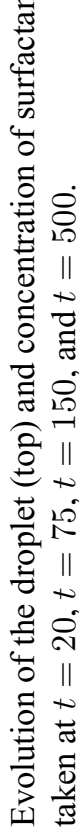

$\ddot{-}$

泀 

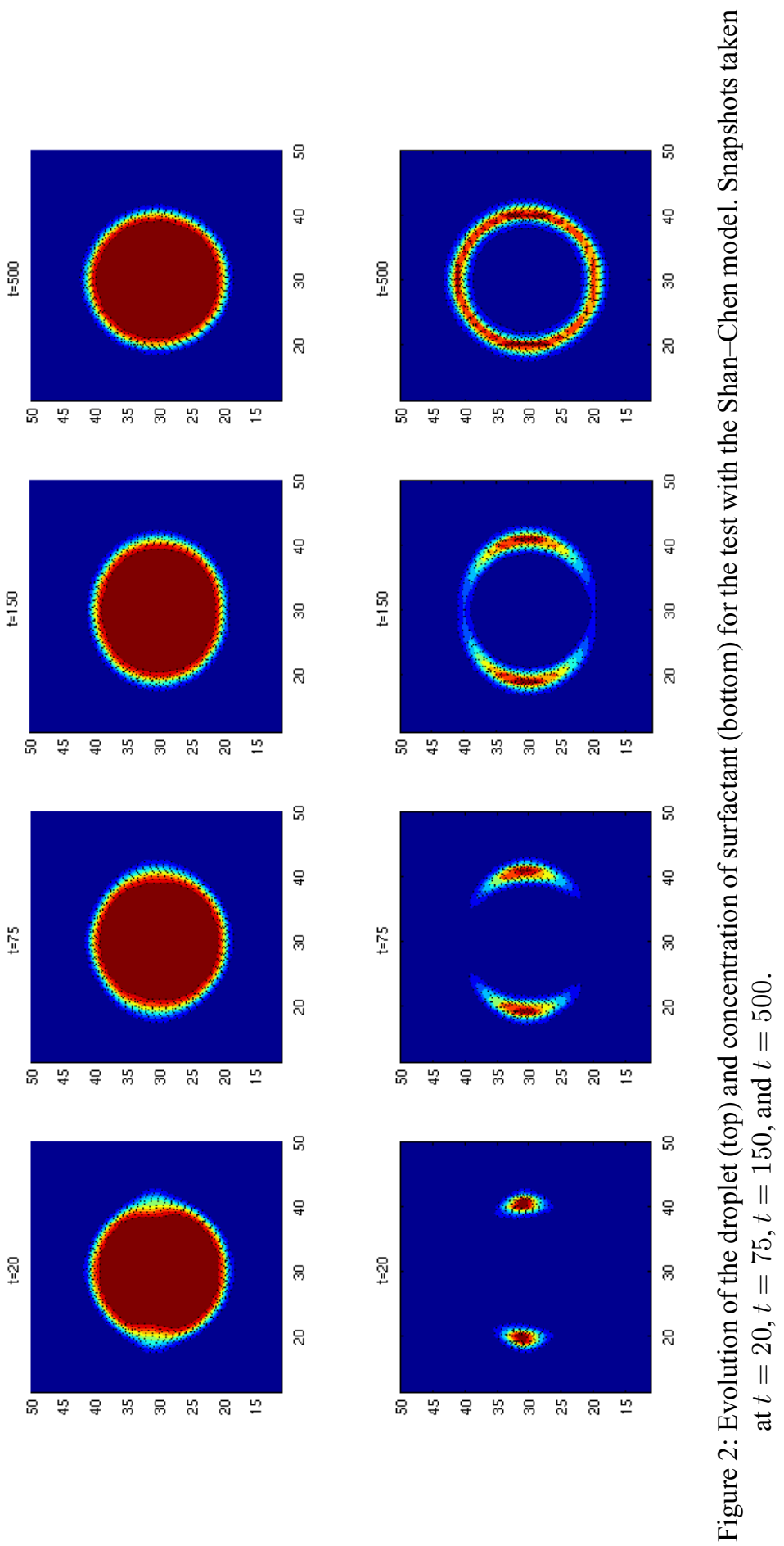
A major difference between the chromodynamic method and the Shan-Chen method is that the former scheme enforces surface tension and phase separation in separate operations, (7) and (8), while these effects are intimately coupled together in the Shan-Chen method through the forcing terms in (9). Therefore, in figure 2 we see diffusion occurring between the red and blue phases once the surfactant is applied.

The final reduction in pressure difference was almost identical to that observed from the chromodynamic model, with the pressure difference dropping from 0.0121 at $t=0$ to 0.0108 at $t=2000$ when the droplet has restabilized.

When the surface tension is weakened at the poles of the droplet, there will be a flow towards the weakened interface, reducing the number of particles at the equator. The result is an elongation along the polar axis and a contraction of the equator [3]. This expected deformation was successfully reproduced by our experiment. Greenspan also observed the droplet's reversion to circular shape with a reduced surface tension as the surfactant diffused over the entire interface.

Neither droplet showed any signs of furrowing or cleavage to the droplet, and the deformation was in both cases fairly small. Although Greenspan's laboratory experiment did show furrowing for certain cases, He and Dembo [4] showed that surface tension variation alone cannot account for such furrowing.

\section{Conclusion}

We have demonstrated ways to implement variable surface tension with both the chromodynamic and Shan-Chen lattice Boltzmann models. Comparing the two models, we think variable surface tension is easier to implement with the chromodynamic model because of the convenient relationship between the surface tension and the parameter $A$ in (7). Implementing variable surface tension with the Shan-Chen model is nonetheless possible if we keep in mind the need to maintain symmetry between sites, and remember that the surface tension vanishes when the concentration exceeds the inequality (14).

The Shan-Chen model is known to possess superior isotropic qualities, and adherence to Laplace's law [26]. Weakening the surface tension in the Shan-Chen method does entail weakening of the phase separation as well, which produces a diffusive interface as observed in figure 2 (top left).

The purpose of our method was to simulate the effects of variable surface tension, not any emulsification process. In order to simulate emulsification, the free energy method is a more circumspect approach, although a more complex representation of the surfactant can be used for the Shan-Chen model, as seen for example in references [27] and [28].

The deformations we observed are relatively small, affirming the conclusions found by He and Dembo [4] who found that surface tension variations alone will generally not yield large deformation or furrowing.

Lattice-Boltzmann methods allow for easy simulation of solutes, a feature which we exploited by implementing the surfactant as a solute. 


\section{Acknowledgements}

The author thanks his advisors Alf Øien and Magne Espedal for helpful discussions and for reviewing the manuscript.

\section{References}

[1] Gunstensen, A.K., Rothmann, D.H., Zaleski, S. \& Zanetti, G., Lattice Boltzmann model of immiscible fluids. Phys Rev A, 43(8), pp. 4320-4328, 1991.

[2] Shan, X. \& Chen, H., Lattice Boltzmann model for simulating flows with multiple phases and components. Phys Rev E, 47(3), pp. 1815-1820, 1993.

[3] Greenspan, H.P., On the Deformation of a Viscous Droplet Caused by Variable Surface Tension. Stud Appl Math, 57(1), pp. 45-58, 1977.

[4] He, X. \& Dembo, M., Numerical simulation of oil-droplet cleavage by surfactant. Journal of Biomechanical Engineering, 118(2), pp. 201-209, 1996.

[5] Greenspan, H.P., On The Dynamics of Cell Cleavage. J theor Biol, 65, pp. 79-99, 1976.

[6] Lubkin, S.R. \& Li, Z., Force and deformation on branching rudiments: cleaving between hypotheses. Biomechan Model Mechanobiol, 1(1), pp. 5$16,2002$.

[7] Orlandini, E., Swift, M. \& Yeomans, J., A Lattice Boltzmann Model of Binary-Fluid Mixtures. Europhys Lett, 32(6), pp. 463-468, 1995.

[8] Lamura, A. \& Gonnella, G., Modeling the Dynamics of amphiphilic fluids. Internat J Modern Phys C, 9(8), pp. 1469-1478, 1998.

[9] Lamura, A., Gonnella, G. \& Yeomans, J., A lattice Boltzmann model of ternary fluid mixtures. Europhys Lett, 45(3), pp. 314-320, 1999.

[10] Theissen, O. \& Gompper, G., Lattice-Boltzmann study of spontaneous emulsification. Eur Phys J B, 11, pp. 91-100, 1999.

[11] Furtado, K., Pooley, C.M. \& Yeomans, J.M., Lattice Boltzmann study of convective drop motion driven by nonlinear chemical kinetics. Phys Rev E, 78, p. 046308, 2008.

[12] Farhat, H. \& Lee, J., Simulation of RBCs Bioconcave shape Using 2D Lattice Boltzmann. Nanotechnology 2008, NSTI Nanotech, volume 3, pp. 433-437, 2008.

[13] Sapir, T. \& Nir, A., A Hydrodynamic Study of the Furrowing Stage During Cleavage. Physicochemical Hydrodynamics, 6, pp. 803-814, 1985.

[14] Li, Z. \& Lubkin, S.R., Numerical analysis of interfacial two-dimensional Stokes flow with discontinuous viscosity and variable surface tension. Int J Numer Meth Fluids, 37, pp. 525-540, 2001.

[15] McNamara, G.R. \& Zanetti, G., Use of the Boltzmann Equation to Simulate Lattice-Gas Automata. Phys Rev Letters, 61(20), pp. 2332-2335, 1988.

[16] He, X. \& Luo, L.S., A priori derivation of the lattice Boltzmann equation. Phys Rev E, 55(6), pp. 6811-6817, 1996. 
[17] Qian, Y., D’Humieres, D. \& Lallemand, P., Lattice BGK Models for NavierStokes Equation. Europhys Lett, 17(6), pp. 479-484, 1992.

[18] Tölke, J., Krafczyk, M., Schulz, M. \& Rank, E., Lattice Boltzmann simulations of binary fluid flow through porous media. Philos Trans $R$ Soc Lond Ser A Math Phys Eng Sci, 360, pp. 535-545, 2002.

[19] Bhatnagar, P.L., Gross, E.P. \& Krook, M., A model for collision processes in gases. i. small amplitude processes in charged and neutral one-component systems. Phys Rev, 94(3), pp. 511-525, 1954.

[20] Reis, T. \& Phillips, T.N., Lattice Boltzmann model for simulating immiscible two-phase flows. J Phys A Math Theor, 40, pp. 4033-4053, 2007.

[21] Latva-Kokko, M. \& Rothman, D.H., Diffusion properties of gradient-based lattice Boltzmann models of immiscible fluids. Phys Rev E, 71, p. 056802, 2005.

[22] Shan, X. \& Doolen, G., Diffusion in a multicomponent lattice Boltzmann equation model. Phys Rev E, 54(4), pp. 3614-3620, 1996.

[23] Marconi, S., Chopard, B. \& Latt, J., Reducing the compressibility of a Lattice Boltzmann fluid using a repulsive force. Internat $J$ Modern Phys $C, \mathbf{1 4 ( 8 )}$, pp. 1015-1026, 2003.

[24] Chin, J., Boek, E.S. \& Coveney, P.V., Lattice Boltzmann simulation of the flow of binary immiscible fluids with different viscosities using the ShanChen microscopic interaction model. Philos Trans $R$ Soc Lond Ser A Math Phys Eng Sci, 360, pp. 547-558, 2002.

[25] Kang, Q., Zhang, D., Chen, S. \& He, X., Lattice Boltzmann simulation of chemical dissolution in porous media. Phys Rev E, 65, 2002.

[26] Hou, S., Shan, X., Zou, Q., Doolen, G.D. \& Soll, W.E., Evaluation of Two Lattice Boltzmann Models for Multiphase Flows. J Comput Phys, 138, pp. 695-713, 1996.

[27] Chen, H., Boghosian, B.M., Coveney, P.V. \& Nekovee, M., A Ternary Lattice Boltzmann Model for Amphiphile Fluids. Proceedings: Mathematical, Physical and Engineering Sciences, 456(2000), pp. 2043-2057, 2000.

[28] Nekovee, M., Coveney, P.V., Chen, H. \& Boghosian, B.M., LatticeBoltzmann model for interacting amphiphilic fluids. Phys Rev E, 62(6), pp. 8282-8294, 2000. 